\title{
18 Cholesterin und Arteriosklerose
}

\author{
(c) Springer-Verlag GmbH Deutschland, ein Teil von Springer Nature 2018 \\ D. Mathias, Fit und gesund von 1 bis Hundert \\ https://doi.org/10.1007/978-3-662-56307-6_18
}

Die Innenauskleidung der Gefäße, das Endothel, ist eine komplizierte Schaltstation, die die Gefäßspannung, die Konzentrierung von Entzündungszellen oder die Gerinnungsabläufe moduliert und dabei Signale aus der Blutzirkulation verwendet. Eine der Ursachen für Fehlfunktionen des Endothels und damit für ein gesteigertes Arterioskleroserisiko sind hohe Spiegel des LDL-Cholesterins. Denn der Versuch der Makrophagen, dieses Low-Density-Lipoprotein-Cholesterin durch oxidative Verdauungsprozesse aus dem Bereich der Gefäßläsionen zu entfernen, geht mit einer permanenten Freisetzung sehr reaktiver Sauerstoffverbindungen einher. Aber diese energiereichen Radikale inaktivieren das vom Endothel gebildete und für die normale Gefäßfunktion bedeutende Stickstoffmonoxid ( $\checkmark$ Kap. 58). Differenziert man hierbei die LDL-Moleküle zusätzlich nach ihrer Größe, dann sind es eher die ganz kleinen und ganz großen Partikel, die besonders risikoreich sind (Grammer et al. 2014).

Die Moleküle der ebenfalls aus vielen Untergruppen bestehenden High-Density-Lipoprotein-Fraktion transportieren Bausteine für die Synthese des Stickstoffmonoxids sowie Botenstoffe, die Entzündungsreaktionen mindern. Eine sehr wichtige Aufgabe dieser HDL-Partikel besteht $u$. a. auch darin, als reverse Cholesterinfähren zur Leber schädliches Cholesterin aus der Zirkulation zu entfernen ( $\triangleright$ Kap. 17). Sie sind dabei in der Lage, die Cholesterinabbauprodukte der in der Arte- rienwand arbeitenden Makrophagen aufzunehmen. Je effektiver dieser Efflux-Prozess verläuft, desto geringer ist die Wahrscheinlichkeit einer Koronarerkrankung, unabhängig von der Höhe der HDLKonzentrationen (Khera et al. 2011).

Hohe Werte des Gesamtcholesterins müssen therapiert werden. Diese Regel gilt aber nicht mehr pauschal für nur mäßig erhöhte Spiegel, denn diese sind nicht generell ein Gesundheitsrisiko. So wurden die bisherigen Empfehlungen der Kardiologen, Cholesterinerhöhungen schon ab 200 mg/dl strikt als behandlungspflichtig anzusehen, korrigiert (Stone et al. 2014; Lloyd-Jones et al. 2014). Nur noch für Patienten mit Herz-Kreislauf-Erkrankungen und Diabetes oder für Personen mit einem statistisch erhöhten Risiko für Herzinfarkt bzw. Schlaganfall und für Menschen mit deutlich erhöhten LDL-Cholesterinwerten über $200 \mathrm{mg} / \mathrm{dl}$ sind Cholesterinsenkungen indiziert.

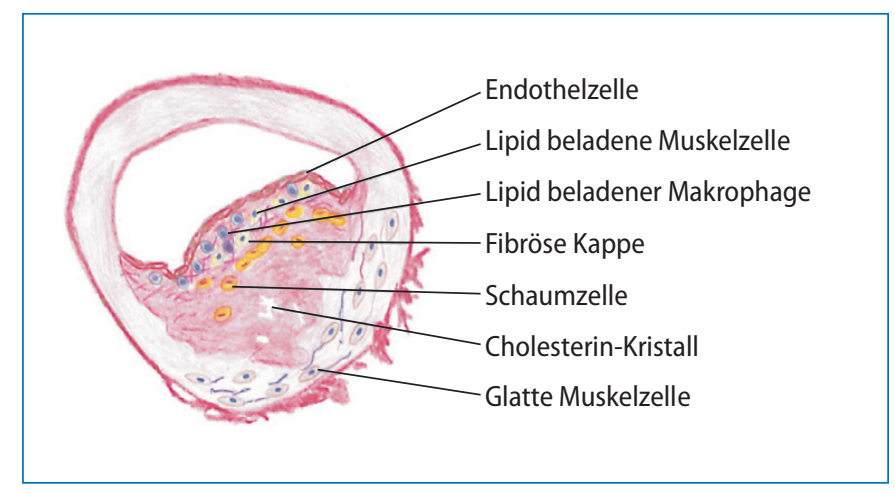

- Abb. 18.1 Gefäßverengung bei Arteriosklerose 